\title{
PRESENTATIONS FOR 3-DIMENSIONAL SPECIAL LINEAR GROUPS OVER INTEGER RINGS
}

\author{
MARSTON CONDER, EDMUND ROBERTSON, AND PETER WILLIAMS
}

(Communicated by Warren J. Wong)

ABSTRACT. The following 2-generator 6-relator presentation is obtained for the 3-dimensional special linear group $\operatorname{SL}\left(3, \mathbb{Z}_{k}\right)$ for each odd integer $k>1$ :

$$
\begin{aligned}
\operatorname{SL}\left(3, \mathbb{Z}_{k}\right) & =\langle x, y| x^{3}=y^{3}=(x y)^{6}=\left(x^{-1} y x^{-1} y^{-1} x y\right)^{2} \\
& =\left(x y^{-1} x y x y^{-1} x^{-1} y^{-1}\right)^{k} \\
& \left.=\left(\left(x y^{-1} x y x y^{-1} x^{-1} y^{-1}\right)^{(k-1) / 2} x y\right)^{4}=1\right\rangle .
\end{aligned}
$$

Alternative presentations for these groups and other groups associated with them are also given.

\section{INTRODUCTION}

The $n$-dimensional special linear group $\operatorname{SL}(n, \mathbb{Z})$ is the multiplicative group of all $n \times n$ matrices with integer entries having determinant 1 . It is well known that $\operatorname{SL}(n, \mathbb{Z})$ is generated by its transvections, that is, by the matrices $T_{i j}$ (for $1 \leq i \neq j \leq n)$ with 1 's on the diagonal and in the $(i, j)$ th position and 0 's elsewhere. In fact if $n \geq 3$, then $\operatorname{SL}(n, \mathbb{Z})$ has a presentation in terms of these generators, with relations as follows (c.f. [6, Corollary 10.3]):

(a) $\left[T_{i j}, T_{k m}\right]=1$ whenever $j \neq k$ and $i \neq m$,

(b) $\left[T_{i j}, T_{j k}\right]=T_{i k}$ whenever $i, j, k$ are distinct, and

(c) $\left(T_{12} T_{21}^{-1} T_{12}\right)^{4}=1$.

In other words, the latter are defining relations for $\operatorname{SL}(n, \mathbb{Z})$ as an abstract group.

If $m$ is any positive integer, the group $\operatorname{SL}\left(n, \mathbb{Z}_{m}\right)$ is similarly defined as the group of all $n \times n$ matrices with entries from the ring $\mathbb{Z}_{m}$ (of all integers modulo $m$ ) and determinant 1 , under matrix multiplication (modulo $m$ ). This is clearly a factor group of $\operatorname{SL}(n, \mathbb{Z})$, obtainable as the image under the homomorphism induced by reduction of integers modulo $m$. The kernel $K_{n, m}$ consists of all those matrices in $\operatorname{SL}(n, \mathbb{Z})$ that are congruent (modulo $m$ ) to the identity matrix, and when $n \geq 3$, the solution of the congruence subgroup problem for $\operatorname{SL}(n, \mathbb{Z})$ given in $[1,5]$ implies that this normal subgroup $K_{n, m}$ is also the smallest normal subgroup of $\operatorname{SL}(n, \mathbb{Z})$ containing the $m$ th power of any

Received by the editors September 7, 1990 and, in revised form, November 20, 1990.

1980 Mathematics Subject Classification (1985 Revision). Primary $20 \mathrm{~F} 05$. 
transvection $T_{i j} \quad(1 \leq i \neq j \leq n)$. In particular, it follows that a presentation for $\operatorname{SL}\left(n, \mathbb{Z}_{m}\right)$ may be obtained by simply adding the single relation $T_{12}^{m}=1$ to those given in (a)-(c) above.

Similar presentations (using elementary matrices and Steinberg relations) for these and other special linear groups are given also in [3, 4].

In this paper several new presentations are obtained in the case where $n=3$, that is, for the groups $\operatorname{SL}\left(3, \mathbb{Z}_{m}\right)$. All use many fewer generators and relations than those previously known, the best being the following 2-generator 6-relator presentation for $\operatorname{SL}\left(3, \mathbb{Z}_{m}\right)$ when $m$ is odd:

$$
\begin{aligned}
\operatorname{SL}\left(3, \mathbb{Z}_{m}\right) & =\langle x, y| x^{3}=y^{3}=(x y)^{6} \\
& =\left(x^{-1} y x^{-1} y^{-1} x y\right)^{2}=\left(x y^{-1} x y x y^{-1} x^{-1} y^{-1}\right)^{m} \\
& \left.=\left(\left(x y^{-1} x y x y^{-1} x^{-1} y^{-1}\right)^{(m-1) / 2} x y\right)^{4}=1\right\rangle ;
\end{aligned}
$$

see Theorem 6. In fact the presentation given in Theorem 6 turns out to be symmetric, in the sense that its two generators can be interchanged by an automorphism; but more importantly, its deficiency (the number of relations minus the number of generators) does not increase with $m$, in contrast to known presentations for other, similar families of finite groups. Also, as a bonus, compact presentations are obtained for finite direct products $\prod_{i} \operatorname{SL}\left(3, p_{i}\right)$, where the $p_{i}$ are distinct primes. Note: $\operatorname{SL}(3, p)$ is $\operatorname{SL}\left(3, \mathbb{Z}_{p}\right)$ when $p$ is prime.

All these presentations are derived rather indirectly from the Steinberg relations (c.f. [6], taking $n=3$ ), and although there seems to be no obvious way to obtain them directly, that of course does not preclude the possibility that similar presentations can be achieved for $\operatorname{SL}\left(n, \mathbb{Z}_{m}\right)$ for more general $n$ and $m$-and indeed this remains an open question.

The impetus for this work comes from a recent discovery made by the first author [2] in the course of tackling a problem to do with trivalent symmetric graphs: the group with presentation

$$
\begin{aligned}
& \langle h, p, q, r, a| h^{3}=a^{2}=p^{2}=q^{2}=r^{2}=1, p q=q p, p r=r p, r q=p q r, \\
& \left.h^{-1} p h=q, h^{-1} q h=p q,(r h)^{2}=1, a^{-1} p a=p, a^{-1} q a=r,(h a)^{12}=1\right\rangle
\end{aligned}
$$

is (somewhat surprisingly) isomorphic to the semidirect product $\operatorname{SL}(3, \mathbb{Z}) \cdot\langle\theta\rangle$, where $\theta$ is the inverse-transpose automorphism of $\operatorname{SL}(3, \mathbb{Z})$. In fact there is an isomorphism taking $h$ and $p$ to the matrices

$$
\left(\begin{array}{lll}
0 & 1 & 0 \\
0 & 0 & 1 \\
1 & 0 & 0
\end{array}\right) \text { and }\left(\begin{array}{rrr}
0 & 1 & 0 \\
1 & 0 & 0 \\
-1 & -1 & -1
\end{array}\right)
$$

respectively, and $a$ to $c \theta$ where $c$ is the matrix

$$
\left(\begin{array}{rrr}
0 & -1 & 0 \\
-1 & 0 & 1 \\
0 & 1 & -1
\end{array}\right)
$$

with the images of $q$ and $r$ determined by the relations $h^{-1} p h=q$ and $a^{-1} q a=r$. Under the given mapping, the transvection $T_{12}$ is the inverse of the image of the element $(h a p)^{4}$, and so immediately we have the following: 
Theorem 1. For every positive integer $m$ the semidirect product $\operatorname{SL}\left(3, \mathbb{Z}_{m}\right) \cdot\langle\theta\rangle$ of $\mathrm{SL}\left(3, \mathbb{Z}_{m}\right)$ by its inverse-transpose automorphism $\theta$ has the presentation

$$
\begin{aligned}
& \langle h, p, q, r, a| h^{3}=a^{2}=p^{2}=q^{2}=r^{2}=1, p q=q p, p r=r p, r q=p q r, \\
& h^{-1} p h=q, h^{-1} q h=p q,(r h)^{2}=1, a^{-1} p a=p, \\
& \left.a^{-1} q a=r,(h a)^{12}=(h a p)^{4 m}=1\right\rangle .
\end{aligned}
$$

This presentation is refined in the next section.

\section{Presentations for $\operatorname{SL}(3, \mathbb{Z})$ AND $\operatorname{SL}\left(3, \mathbb{Z}_{m}\right)$}

In the presentation given in the preceding theorem, the generators $q$ and $r$ are obviously redundant, and also many of the relations can be replaced by simpler ones or eliminated.

For example, the relation $r q=p q r$ can be seen to be a consequence of the others, as follows: $p q r=p h^{-1} p h r=p h^{-1} p r h^{-1}=p h^{-1} r p h^{-1}=p r h p h^{-1}=$ $p r h^{-2} p h^{2}=p r p q=r q$. Similarly the relation $h^{-1} q h=p q$ gives $(p h)^{3}=$ $p h^{-2} p h^{2} h^{-1} p h=p(p q) q=p^{2} q^{2}=1$, and then both $h^{-1} q h=p q$ and $p q=q p$ can be replaced by the single relation $(p h)^{3}=1$, for this relation implies $h^{-1} q h=h^{-2} p h^{2}=h p h h=p h^{-1} p h=p q$, and also $q p q=h^{-1} p h p h^{-1} p h=$ $h(h p h p h) h p h=h p h p h=p$. The consequent elimination of the redundant generators $q$ and $r$ via $q=h^{-1} p h$ and $r=a^{-1} q a=a^{-1} h^{-1} p h a$ gives $\left(a h^{-1} \text { phah }\right)^{2}=1$ in place of $(r h)^{2}=1$, and $\left(p a h^{-1} p h a\right)^{2}=1$ in place of $p r=r p$, but then since $\left(p a h^{-1} p h a\right)^{2}=\left(a^{-1} p h^{-1} p h a\right)^{2}=\left(a^{-1} h p h^{-1} a\right)^{2}=$ $a^{-1} h p^{2} h^{-1} a$, the second of these new relations is redundant as well. Thus we obtain the following presentation for $\operatorname{SL}(3, \mathbb{Z}) \cdot\langle\theta\rangle$ :

$$
\left\langle h, p, a \mid h^{3}=a^{2}=p^{2}=(p h)^{3}=\left(a h^{-1} p h a h\right)^{2}=(a p)^{2}=(h a)^{12}=1\right\rangle,
$$

and correspondingly, the following presentation for $\operatorname{SL}\left(3, \mathbb{Z}_{m}\right) \cdot\langle\theta\rangle$ whenever $m>0$ :

$$
\left\langle h, p, a \mid h^{3}=a^{2}=p^{2}=(p h)^{3}=\left(a h^{-1} p h a h\right)^{2}=(a p)^{2}=(h a)^{12}=(h a p)^{4 m}=1\right\rangle .
$$

Note that the inverse-transpose automorphism $\theta$ is effectively the same for both $\operatorname{SL}(3, \mathbb{Z})$ and $\operatorname{SL}\left(3, \mathbb{Z}_{m}\right)$, having order 2 and normalizing the special linear group in each case.

Next let $x=h$ and $y=a h a$, and (just for aesthetics) also let $z=p$. The subgroup $N$ generated by these three elements has index 2 in each of the above finitely-presented groups, with $N h=N, N p=N,(N a) h=(N a h a) a=N a$, and $(N a) p=(N p) a=N a$. Clearly $\{1, a\}$ is a Schreier transversal for $N$, and then $x, y$, and $z$ can be taken as Schreier generators for $N$, with the Reidemeister relations obtained as the conjugates of the appropriate original relations by the element $a$ in each case. Specifically, we now have the following:

Theorem 2. The group $\operatorname{SL}(3, \mathbb{Z})$ has presentation

$$
\left\langle x, y, z \mid x^{3}=y^{3}=z^{2}=(x z)^{3}=(y z)^{3}=\left(x^{-1} z x y\right)^{2}=\left(y^{-1} z y x\right)^{2}=(x y)^{6}=1\right\rangle \text {. }
$$

Theorem 3. For every positive integer $m$, the group $\operatorname{SL}\left(3, \mathbb{Z}_{m}\right)$ has presentation

$$
\begin{aligned}
\langle x, y, z| x^{3} & =y^{3}=z^{2}=(x z)^{3}=(y z)^{3} \\
& \left.=\left(x^{-1} z x y\right)^{2}\left(y^{-1} z y x\right)^{2}=(x y)^{6}=(x z y z)^{2 m}=1\right\rangle .
\end{aligned}
$$


$\left(\right.$ Note that $(h a p)^{2}=$ haphap $=$ hpahap $=x z y z$, and then also $a^{-1}(h a p)^{2} a=$ $y z x z$, which is conjugate to $x z y z$ within the subgroup $N$.)

The 3-generator 9-relator presentation given in Theorem 2 is a refinement of the one given in Corollary $1(\mathrm{~b})$ in [2], and is perhaps the best possible that can be obtained for $\operatorname{SL}(3, \mathbb{Z})$ using this approach. On the other hand, Theorem 3 can be further improved, as we shall see.

One way to continue is to examine the relation $(x z y z)^{2 m}=1$ more closely.

First, the relations we have imply $(x z y z)^{4}=x y x^{-1} y^{-1} x^{-1} y x^{-1} y$. One nice way to see this is to use the following consequences of the original relations as a set of rewriting rules:

$$
\begin{array}{lll}
p a=a p, & q a=a r, & r a=a q, \\
p h=h q, & q h=h p q, & r h=h^{-1} r, \\
p h^{-1}=h^{-1} p q, & q h^{-1}=h^{-1} p, & r h^{-1}=h r ;
\end{array}
$$

for then

$$
\begin{aligned}
(x z y z)^{4} & =(\text { hpahap })^{4}=\text { haphap }(\text { hap })^{6}=\text { hahqap }(\text { hap })^{6}=\text { haharphap }(\text { hap })^{5} \\
& =\text { hahah }^{-1} \text { rqap }(\text { hap })^{5}=\text { hahah }^{-1} \text { aqrphap }(h a p)^{4}, \text { and so on, }
\end{aligned}
$$

eventually giving

$$
(x z y z)^{4}=h_{a h a h^{-1}} a h^{-1} a h^{-1} a h a h^{-1} a h a=x y x^{-1} y^{-1} x^{-1} y x^{-1} y .
$$

The same sort of calculation also shows that

$$
(x z y z)^{2}=(\text { hpahap })^{2}=h_{a h a p h}-1 a h a=x y z x^{-1} y,
$$

although this can also be obtained directly as follows:

$$
\begin{aligned}
(x z y z)^{2} & =\left(x y^{-1} z y^{-1}\right)^{2}=x y^{2} z y^{-1}\left(x y^{-1} z y\right) y=x y^{2} z y^{-1}\left(y^{-1} z y x^{-1}\right) y \\
& =x y(y z y z y) x^{-1} y=x y z x^{-1} y,
\end{aligned}
$$

using only the relations given in the presentation of Theorem 3 .

Now if $m$ is odd, say $m=2 j+1$, the relation $(x z y z)^{2 m}=1$ becomes $(x z y z)^{4 j+2}=1$ and thereby

$$
1=\left((x z y z)^{4}\right)^{j}(x z y z)^{2}=\left(x y x^{-1} y^{-1} x^{-1} y x^{-1} y\right)^{j} x y z x^{-1} y,
$$

which in turn gives

$$
z=z^{-1}=x^{-1} y\left(x y x^{-1} y^{-1} x^{-1} y x^{-1} y\right)^{j} x y=\left(x^{-1} y x y x^{-1} y^{-1} x^{-1} y\right)^{j} x^{-1} y x y .
$$

In other words, when $m$ is odd the generator $z$ is redundant, and therefore, can be eliminated. Thus we have (ignoring the trivial case where $m=1$ ):

Theorem 4. For every odd integer $k>1$ the group $\operatorname{SL}\left(3, \mathbb{Z}_{k}\right)$ has presentation

$$
\begin{aligned}
\langle x, y| x^{3}=y^{3}=(x y)^{6} & =\left(\left(x^{-1} y x y x^{-1} y^{-1} x^{-1} y\right)^{(k-1) / 2} x^{-1} y x y\right)^{2} \\
& =\left(\left(x^{-1} y x y x^{-1} y^{-1} x^{-1} y\right)^{(k-1) / 2} x^{-1} y x y x\right)^{3} \\
& =\left(\left(x^{-1} y x y x^{-1} y^{-1} x^{-1} y\right)^{(k-1) / 2} x^{-1} y x y^{-1}\right)^{3} \\
& =\left(\left(x^{-1} y x y x^{-1} y^{-1} x^{-1} y\right)^{(k-1) / 2} x^{-1} y x y x y x^{-1}\right)^{2} \\
& \left.=\left(\left(x^{-1} y x y x^{-1} y^{-1} x^{-1} y\right)^{(k-1) / 2} x^{-1} y x y^{-1} x y^{-1}\right)^{2}=1\right\rangle .
\end{aligned}
$$


This 2-generator 8-relator presentation (obtainable using Tietze transformations from the one in Theorem 3) is rather complicated; a much improved version is given later.

\section{DiRECT PRODUCTS}

In this section we recall the solution to the congruence subgroup problem for $\operatorname{SL}(n, \mathbb{Z})$ when $n \geq 3$ (see [1] or [5]): if $m$ is any positive integer, then the subgroup $K_{n, m}$ of all matrices that are congruent modulo $m$ to the identity matrix $I_{n}$ is also the smallest normal subgroup of $\operatorname{SL}(n, \mathbb{Z})$ containing the $m$ th power of any transvection $T_{i j}(1 \leq i \neq j \leq n)$.

Now if $k$ and $m$ are coprime positive integers, clearly $K_{n, k} \cap K_{n, m}=K_{n, k m}$; while on the other hand, as $1=k u+m v$ for some $u, v \in \mathbb{Z}$, the product $K_{n, k} K_{n, m}$ contains every transvection: $T_{i j}=T_{i j}^{k u+m v}=\left(T_{i j}^{k}\right)^{u}\left(T_{i j}^{m}\right)^{v} \in$ $K_{n, k} K_{n, m}$ whenever $1 \leq i \neq j \leq n$; and therefore, $K_{n, k} K_{n, m}=\operatorname{SL}(n, \mathbb{Z})$. In particular, this means the factor group $\operatorname{SL}(n, \mathbb{Z}) / K_{n, k m}$ is isomorphic to the direct product of its subgroups $K_{n, k} / K_{n, k m}$ and $K_{n, m} / K_{n, k m}$; but then because $K_{n, k} / K_{n, k m}=K_{n, k} /\left(K_{n, k} \cap K_{n, m}\right) \cong\left(K_{n, k} K_{n, m}\right) / K_{n, m}=\mathrm{SL}(n, \mathbb{Z}) / K_{n, m}$ and similarly $K_{n, m} / K_{n, k m} \cong \mathrm{SL}(n, \mathbb{Z}) / K_{n, k}$, the quotient $\mathrm{SL}(n, \mathbb{Z}) / K_{n, k m}$ is isomorphic to the direct product of the individual factors $\operatorname{SL}(n, \mathbb{Z}) / K_{n, k}$ and $\operatorname{SL}(n, \mathbb{Z}) / K_{n, m}$.

The following consequence is almost immediate:

Theorem 5. If $k_{1}, k_{2}, \ldots, k_{s}$ are pairwise coprime positive integers whose prod$u c t$ is $m$, then the direct product $\prod_{1 \leq i \leq s} \operatorname{SL}\left(3, \mathbb{Z}_{k_{i}}\right)$ has presentation

$$
\begin{aligned}
\langle x, y, z| x^{3}=y^{3}=z^{2}=(x z)^{3}=(y z)^{3}=\left(x^{-1} z x y\right)^{2} \\
\left.=\left(y^{-1} z y x\right)^{2}=(x y)^{6}=(x z y z)^{2 m}=1\right\rangle .
\end{aligned}
$$

This can be proved by extending the above argument to show $\operatorname{SL}(n, \mathbb{Z}) / K_{n, m}$ is isomorphic to the direct product of the individual factors $\operatorname{SL}(n, \mathbb{Z}) / K_{n, k_{i}}$ for $1 \leq i \leq s$ (and any $n \geq 3$ ), and then applying the results of $\S 2$.

\section{A COMPACT SYMMETRIC PRESENTATION FOR $\operatorname{SL}\left(3, \mathbb{Z}_{k}\right)$}

If $k$ is an odd integer greater than 1, Theorem 5 gives this 3-generator 9relator presentation for the direct product $\operatorname{SL}(3,2) \times \operatorname{SL}\left(3, \mathbb{Z}_{k}\right)$ :

$$
\begin{aligned}
\langle x, y, z| x^{3}=y^{3}= & z^{2}=(x z)^{3}=(y z)^{3}=\left(x^{-1} z x y\right)^{2} \\
& \left.=\left(y^{-1} z y x\right)^{2}=(x y)^{6}=(x z y z)^{4 k}=1\right\rangle .
\end{aligned}
$$

Again $(x z y z)^{4}=x y x^{-1} y^{-1} x^{-1} y x^{-1} y$ is implied by the other relations, so the final relation can be replaced by $\left(x y x^{-1} y^{-1} x^{-1} y x^{-1} y\right)^{k}=1$ if necessary. On the other hand, the third generator $z$ cannot be eliminated, for the subgroup $L$ generated by $x$ and $y$ in this group has index 8: its right cosets may be taken as $L, L z, L z x, L z y, L z x z, L z y z, L z x z y$, and $L z y z x$, or (in terms of our earlier generators) as $L, L p, L q, L r, L p q, L p r, L q r$, and $L r q$ respectively. These cosets are permuted (under right multiplication) by the generators $x, y$, 
and $z$ as follows:

$x$ induces the permutation $(L z, L z x, L z x z)(\operatorname{Lzy} z, \operatorname{Lzy} z x, L z x z y)$, $y$ induces the permutation $(L z, L z y, L z y z)(L z x z, L z x z y, L z y z x)$, and

$z$ induces the permutation $(L, L z)(L z x, L z x z)(L z y, L z y z)(L z x z y, L z y z x)$; for example, the relations $x^{3}=y^{3}=z^{2}=(x z)^{3}=(y z)^{3}=\left(x^{-1} z x y\right)^{2}=$ $\left(y^{-1} z y x\right)^{2}=1$ give

$$
\begin{aligned}
(\operatorname{Lzxzy}) x & =\operatorname{Lzx}\left(z y x y^{-1}\right) y=\operatorname{Lzx}\left(y x^{-1} y^{-1} z\right) y \\
& =L\left(z x y x^{-1}\right) y^{-1} z y=L\left(x y^{-1} x^{-1} z\right) y^{-1} z y \\
& =\left(L x y^{-1} x^{-1}\right) z y^{-1} z y=L z y^{-1} z y=L y z y y=L y^{-1} z y^{-1}=L z y z,
\end{aligned}
$$

although this calculation is much easier using the earlier generators.

These permutations can also be checked using the Todd-Coxeter enumeration process, and the interested reader may even wish to verify by computer (using CAYLEY for instance) that they generate a group of order 168 , isomorphic to $\operatorname{SL}(3,2)$.

More importantly, this permutation representation of $\operatorname{SL}(3,2) \times \operatorname{SL}\left(3, \mathbb{Z}_{k}\right)$ on cosets of the subgroup $L$ can now be used to obtain a much neater presentation of $\operatorname{SL}\left(3, \mathbb{Z}_{k}\right)$.

First, using $\{1, z, z x, z y, z x z, z y z, z x z y, z y z x\}$ as transversal, the Reidemeister-Schreier process gives the following presentation for the subgroup $L$ :

$$
\left\langle x, y \mid x^{3}=y^{3}=(x y)^{6}=\left(x^{-1} y x^{-1} y^{-1} x y\right)^{2}=\left(x y^{-1} x y x y^{-1} x^{-1} y^{-1}\right)^{k}=1\right\rangle .
$$

Note that all the Schreier generators are easily expressible as words in $x$ and $y$; for instance,

$$
\begin{aligned}
(z y z x) x(z x z y)^{-1} & =z y z\left(x^{-1} y^{-1} z\right) x^{-1} z=z y z\left(y^{-1} z y x y^{-1}\right) x^{-1} z \\
& =(z y z) y^{-1} z y\left(x y^{-1} x^{-1} z\right) \\
& =\left(y^{-1} z y^{-1}\right) y^{-1} z y\left(z x y x^{-1}\right)=y^{2} z y z y z x y x^{-1}=y x y x^{-1} .
\end{aligned}
$$

Also many of the relators provided by the Reidemeister-Schreier process are conjugates or inverses of each other. For instance $\left(x y x^{-1} y^{-1} x^{-1} y x^{-1} y\right)^{k}$ is the inverse of $\left(y^{-1} x y^{-1} x y x y^{-1} x^{-1}\right)^{k}$, which in turn is obviously a conjugate of $\left(x y^{-1} x y x y^{-1} x^{-1} y^{-1}\right)^{k}$.

On the other hand, the matrices given in $\S 1$ (when taken together with the subsequent definitions $x=h$ and $y=a h a$ ), provide us with a concrete faithful matrix representation of the group $L$ over the ring $\mathbb{Z}_{2 k}$ : as representatives of the generators $x$ and $y$ of $L$ we may define the matrices

$$
X=\left(\begin{array}{lll}
0 & 1 & 0 \\
0 & 0 & 1 \\
1 & 0 & 0
\end{array}\right) \text { and } Y=\left(\begin{array}{rrr}
1 & 0 & 1 \\
0 & -1 & -1 \\
0 & 1 & 0
\end{array}\right)
$$

respectively, treating their entries as integers modulo $2 k$. In particular, these matrices satisfy the relations of the presentation obtained for $L$ above, with 


$$
X Y^{-1} X Y X Y^{-1} X^{-1} Y^{-1}=\left(\begin{array}{lll}
1 & 0 & 2 \\
0 & 1 & 0 \\
0 & 0 & 1
\end{array}\right)
$$

the square of the transvection $T_{13}$.

Now consider the matrix $W=\left(X Y^{-1} X Y X Y^{-1} X^{-1} Y^{-1}\right)^{(k-1) / 2} X Y$. Clearly

$$
W=\left(\begin{array}{ccc}
1 & 0 & k-1 \\
0 & 1 & 0 \\
0 & 0 & 1
\end{array}\right)\left(\begin{array}{rrr}
0 & -1 & -1 \\
0 & 1 & 0 \\
1 & 0 & 1
\end{array}\right)=\left(\begin{array}{rrr}
k-1 & -1 & k-2 \\
0 & 1 & 0 \\
1 & 0 & 1
\end{array}\right),
$$

and then, modulo $2 k$ remember,

$$
W^{4}=\left(\begin{array}{ccc}
k+1 & k & k \\
0 & 1 & 0 \\
k & 0 & 1
\end{array}\right)
$$

which has order 3 , and is also easily seen to be congruent to the identity modulo $k$. Next, $W^{4}$ and its conjugate $X^{-1} W^{4} X$ together generate a Frobenius group of order 21: for

$$
\left(W^{4}\right)^{-1} X^{-1} W^{4} X=\left(\begin{array}{ccc}
k+1 & k & 0 \\
k & k+1 & k \\
0 & k & 1
\end{array}\right),
$$

which has order 7 and is conjugated to its 4 th power by each of $W^{4}$ and $X^{-1} W^{4} X$. Moreover, this subgroup is normalized by each of $X$ and $Y$ : putting $U=\left(W^{4}\right)^{-1} X^{-1} W^{4} X$, we find by straightforward calculation that $X^{-1}\left(X^{-1} W^{4} X\right) X=U^{-1} W^{4}$ and $Y^{-1} W^{4} Y=U^{3} W^{4}$ and $Y^{-1}\left(X^{-1} W^{4} X\right) Y=$ $W^{4}$; indeed also $X^{-1} U X=Y^{-1} U Y=U^{2}$ and $X^{-1} W^{4} X=U^{2} W^{4}$.

Correspondingly, in $L$ the element $\left(\left(x y^{-1} x y x y^{-1} x^{-1} y^{-1}\right)^{(k-1) / 2} x y\right)^{4}$ and its conjugate by $x$ generate a normal subgroup $F$ of order 21 , identifiable with any subgroup of index 8 in the group $\operatorname{SL}(3,2)$, and then $L$ is a direct product of $F$ with an isomorphic copy of $\operatorname{SL}\left(3, \mathbb{Z}_{k}\right)$. In particular, adjoining the relation $\left(\left(x y^{-1} x y x y^{-1} x^{-1} y^{-1}\right)^{(k-1) / 2} x y\right)^{4}=1$ to the presentation found earlier for $L$ must give a presentation for $\operatorname{SL}\left(3, \mathbb{Z}_{k}\right)$ alone.

Thus we have:

Theorem 6. If $k$ is any odd integer greater than 1, the 3-dimensional special linear group $\operatorname{SL}\left(3, \mathbb{Z}_{k}\right)$ over the ring $\mathbb{Z}_{k}$ of integers modulo $k$ has presentation

$$
\begin{aligned}
\langle x, y| x^{3}=y^{3}=(x y)^{6}=\left(x^{-1} y x^{-1} y^{-1} x y\right)^{2} & =\left(x y^{-1} x y x y^{-1} x^{-1} y^{-1}\right)^{k} \\
& \left.=\left(\left(x y^{-1} x y x y^{-1} x^{-1} y^{-1}\right)^{(k-1) / 2} x y\right)^{4}=1\right\rangle .
\end{aligned}
$$

In particular, when $k=p$ for some odd prime $p$, the latter is a presentation of the group $\mathrm{SL}(3, p)$ and more generally if $k$ is the product of distinct odd primes $p_{1}, p_{2}, \ldots, p_{s}$, it is a presentation of the direct product $\prod_{1 \leq i \leq s} \operatorname{SL}\left(3, p_{i}\right)$.

Finally we note that this presentation is symmetric, in the sense that the generators $x$ and $y$ may be interchanged by an automorphism of the group. Equivalently, those relations that are obtained by interchanging occurrences of $x$ and $y$ in the given relations are also satisfied, and this is an immediate consequence of the fact that the matrix representatives $X$ and $Y$ considered above are conjugates of each other by $c \theta$, where $\theta$ is the inverse-transpose automorphism and $c$ is the matrix given in the introduction. 


\section{ACKNOWLEDGMENTS}

The discovery of these presentations resulted from mutual discussions between the three authors at the Groups-St. Andrews 1989 conference, and subsequent calculations performed with the aid of the CAYLEY system. The first author is grateful to the following bodies for research support: the Royal Society of New Zealand, the N. Z. Lotteries Board, and the N. Z. University Grant Committee.

\section{REFERENCES}

1. H. Bass, M. Lazard, and J-P. Serre, Sous-groupes d'indice fini dans $\operatorname{SL}(n, Z)$, Bull. Amer. Math. Soc. 70 (1964), 385-392.

2. Marston Conder, A surprising isomorphism, J. Algebra 129 (1990), 494-501.

3. Sherry M. Green, Generators and relations for the special linear group over a division ring, Proc. Amer. Math. Soc. 62 (1977), 229-232.

4. Jürgen Hurrelbrink, On presentations of $\mathrm{SL}_{n}\left(\mathbb{Z}_{s}\right)$, Comm. Algebra 11 (1983), 937-947.

5. Jens Mennicke, Finite factor groups of the unimodular group, Ann. of Math. (2) 81 (1965), 31-37.

6. John Milnor, Introduction to algebraic K-theory, Ann. of Math. Stud., no. 72, Princeton Univ. Press, Princeton, NJ, 1971.

Department of Mathematics \& Statistics, University of Auckland, Private Bag, AuckLAND, New Zealand

Mathematical Institute, University of St. Andrews, St. Andrews, KY16 9SS, ScotLAND

Department of Mathematics, California State University, San Bernardino, CaliforNIA 92407 